\title{
Basic of Laser and its Progress in Different Fields
}

\author{
Jyoti Kattimani \\ Assistant Professor of Physics, Government First Grade College Manvi, Karanataka India
}

\begin{abstract}
The word LASER stands for Light Amplification by Stimulated Emission of Radiation. It has became so familiar to everyone and made essential in everyday life. During these days LASER technology has made great progress due to its multiple applications. The paper describes the history of the laser, characteristics, classification based on wavelength, different types of laser and its application in different fields. Current applications were lasers are used in space communication.
\end{abstract}

Keywords: Laser, Maser

\section{Introduction}

The acronym LASER stands for Light Amplification by Stimulated Emission of Radiation, these day's become so common and popular in everyday life that it is now termed as laser. The fundamental theories of lasers, its historical development from milliwatts to pentawatts in terms of power, beam characteristics, principles and their applications.

In 1900 Max Plank provided the understanding that light is a form of electromagnetic radiation. The principle of the laser was first given by Albert Einstein using Plank's law of radiation that was based on Einstein coefficients or probability coefficients for absorption, spontaneous and emission of electromagnetic radiation. However, it was not until the late 1940s that engineers began to utilize this principle for practical purposes.

In 1960 the first working laser was built by Theodore Maiman at Hughes Research lab. The laser was a solid state ruby laser based on optical pumping. The phenomenon of stimulated emission and negative absorption was confirmed by R. W. Ladenburg in 1928. However in 1947, Willis E Lamb and R. C. Rutherford found apparent stimulated emission in hydrogen spectra and effected the first demonstration of stimulated emission. Alfred Kastler proposed the method of optical pumping in 1950, experimentally confirmed, two years later by Brossel, Kastler and Winter.

In 1952, Ch. Towns, J. Gordon and H. Zeiger in U. S. AN. Basov and A. Prokhorovin USSR, independently suggested the principle of generating and amplifying microwave oscillations based on the concept of stimulated radiation. This leads to the invention of Microwave Amplification by Stimulated Emission of Radiation i.e. MASER in 1954. The two-level system was used in MASER. Basov and Prokhonov in 1955 suggested the use of three-level system. Townes and Schawlow and Basov and Prokhorov in 1955 independently expressed their ideas about extending the concept of MASER to optical frequencies. They further developed the concept of an optical amplifier surrounded by an optical mirror resonant cavity to allow for the growth of the beam. They received the Nobel Prize for their work in this field.

\section{Characteristics}

The emitted laser has four different characteristic features

1) Monochromatic: in which all waves have same frequency and energy.

2) Coherent: all waves are in constant phase relation and are related to each other, both in speed and time.

3) Intensity: extreme intensity is observed in a very small spot size.

4) Focus: it is highly directional beam, does not spread out like a ordinary beam of light.

\section{Classification}

Lasers are classified according to the spectrum of light and material used. The characteristic of a laser depends on its wavelength. Different wavelengths are classified into three groups:

1) The UV range ultra spectrum approximately 400-700nm.

2) The VIS range visible spectrum approximately 400$700 \mathrm{~nm}$.

3) The IR range infra-red spectrum approximately 700nm.

The light given out by carbon dioxide lasers and neodymium lasers cannot be seen by the human eye due the infrared region of the spectrum. However the red light given out by the ruby laser or helium- neon laser, blue and green light given out by the argon laser, can be seen by human eye. But light given out by krypton-flouride lasers lies in the ultra violet region and cannot be directly detected visually.

A laser consists of a cavity with mirror at each end and a gain medium, placed between the two mirrors. Excited by an energy source, the medium emits light which circulates between the mirrors and is amplified at each passage. One of the mirrors is slightly transparent to enable the laser beam to exit from the cavity. Lasers can be distinguished according to the nature of the amplifying medium: gas, liquid or solid [6-7].

\section{Types of laser}

1) Solid state lasers: Ruby (Cr: Al2O3) laser, Nd-YAG (Nd: Y3Al5O12, Neodymium doped yttrium aluminium garnet) laser, Nd-glass laser.

2) Gas lasers: He-Ne laser, $\mathrm{CO} 2$ laser, argon ion laser.

3) Liquid lasers: dye lasers (Organic substances dissolved in water, ethyl alcohol, methanol, ethylene glycol etc.) 


\section{International Journal of Science and Research (IJSR) \\ ISSN (Online): 2319-7064}

Index Copernicus Value (2013): 6.14 | Impact Factor (2015): 6.391

4) Excimer lasers: Excimers are molecules which are bound in their excited electronic states but are unstable in their ground states e.g., Argon fluoride, krypton chloride, Krf, $\mathrm{XeBr}$ etc.

5) Semiconductor lasers: Also called junction laser or diode laser and today very important type of lasers having its applications in fibre optics communication.

\section{Application of laser in different Field}

Industrial applications: laser welding, drilling, annealing, sputtering, cutting such as glass decoration, trimming, milling etc. the most advantage of laser operations contains machining the product without any mechanical contact for example remote machining or machining in a protective atmosphere, in machining parts of the product difficult to access, as well as in technological treatment of materials that cannot be effected by classical methods.. Since last 25 years, the publishing and newsprint industries have been revolutionized by the use of lasers, which have replaced traditional "hot metal" printing. Other everyday laser uses include barcode readers, laser pointers as well as laser printers etc.

Medicine: laser used in ophthalmology it is used in the treatment of myopia and cataract where they enable patients with visual impairment to regain a clear vision and forget about the stress of wearing glasses or contact lens. In dermatology used for the treatment of vascular lesions such as angiomas, telangiectasias, spider naevi, pigmented lesions ( brown spots, freckles, naevus of ota) etc. Laser surgery has a advantages of reducing the risk of infection and it promotes healing. Laser is used in cosmetic surgery to erase cellulite and superficial wrinkles etc. laser are also used in dentistry for gum care and treatment of tooth decay etc.

Military field: lasers are used in various military applications, the most widely used is a laser range finders it can measure with great accuracy the target distance and to obtain the optimal trajectory of a missile and higher reliability of the hit. It is used as laser markers in guns to identify the target at a distance of up to $20 \mathrm{~m}$. Laser defense systems can detect enemy ships and missiles while at the same time disabling them.

Communication: Radiations of laser are used in mass communications such as telephone conversations, television channels and space communication system. Because of its directionality it can be send to the longer distances. So laser radiations are very much useful for the free space satellite laser communication. Different technologies are created by the NASA which uses laser communications. One of recent development is NASA accomplishment was a successful carried the laser pulses with the MESSENGER spacecraft and an Earth-based observatory which is new development for laser transmission in space region. Satellite-to-satellite communication using the laser communication was developed by the NASA.

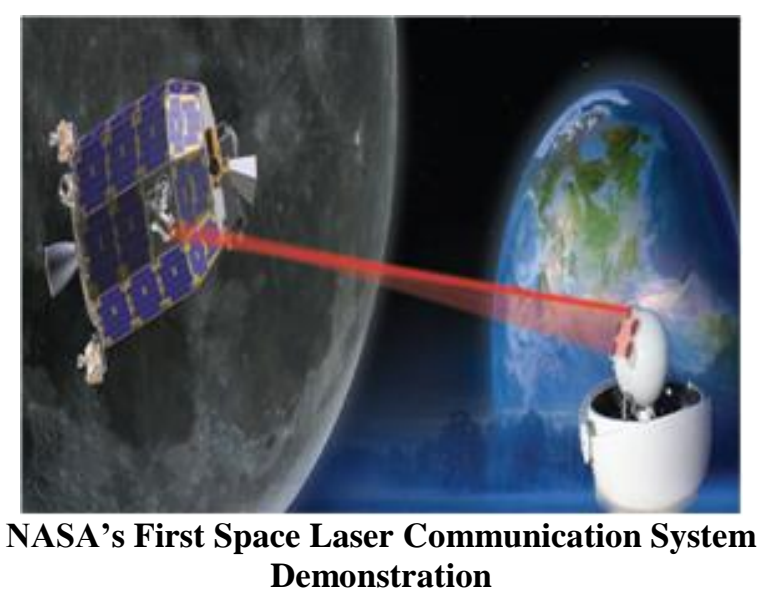

\section{Conclusion}

The aim of the present paper is to describe about the laser technology that has made rapid progress over the past few decades. Due to its many advantages, it has been widely used in different fields such as industrial area, medical science, military field and one of most important and current application these are used in space communication form this we can conclude that laser plays an important role in the society.

\section{References}

[1] Wilson, J. and Hawkes, J.F.B. Lasers: Principles and Applications, prentice Hall Publications (eds) (1987) ISBN: 013523697-5.

[2] O'Shea, D.C., Callen, W.R., and Rhodes, W.T. Introduction to Lasers and their Applications, AddisonWesley Publishing Company, Inc., Philippines. (eds) (1977) ISBN: 020105509-0.

[3] Ifflander, R. Solid State Laser Material Processing: Fundamental Relations \& Technical Realizations, Springer-Verlag, Berlin, Heidelberg. (ed.) (2001) ISBN: 354066980-9.

[4] Letokhov, V.S., Shank, C.V., Shen, Y.R., and Walther, H. Interaction of Intense Laser Light with Free Electrons, M.V. Fedorov; Laser Science and Technology and International Handbook, Harwood Academic Publishers GmbH, Switzerland. (eds) (1991) ISBN-3718651262.

[5] Weber, M.J. Handbook of Laser Science and Technology, CRC Press, Boca Raton, FL, Ann Arbor, MI, Boston, MA. (ed.) (1991) ISBN: 084933506-X.

[6] S. Nagabhushana and N. Sathyanarayana, Laser \& Optical Instrumentation I. K. International Publishing (2010) ISBN-978-93-80578-23-1

[7] C. Breck Hitz, J. Ewing, Jeff Hecht, (2012) Introduction to Laser Technology, Fourth Edition, IEEE PressWiley.

[8] Karlf. Renk, (2012) Basis of Laser Physics for Students of Science \& Engineering, Springer.

[9] K. R. Nambiar, (2004) Lasers Principles, Types \& Applications, New Age International Publishers. 